\title{
Programming and Implementation of Assessment
}

\section{Nermin Ademi*}

Department of Business and Economics, Ministry of Culture, Macedonia

\begin{abstract}
The process of assessment is a continuous process which is periodically being analyzed and planned for the efficiency of labor wherewith it enables achievement of successful strategic goals of the enterprise. The basis goal of assessment is study from the past and improvement of the future. To assess means to collect information about the outcome of the labor, about the behavior at work, to analyze these information, to identify the criteria and the indicators for assessment in order to support the feedback loop with the employees. The essence of assessment comprises of measurement and assessment of the outcomes and the manner how people achieved that, in order to create conditions for development of rightful awarding. Today the process of implementation of assessment in many developed countries is treated as an integral part in the field of development of policy of human potential.
\end{abstract}

Keywords: Assessment; Methods; Set-up; Efficiency

\section{Introduction}

\section{Assessment and the need of its implementation}

The development of a system of assessment of successfulness should be an important segment in the managerial action. Assessment of employees is an important task of management, because it should be able to undertake administrative decisions which cause changes in the employee. The management should have the current information about the successfulness of the units and the sectors. Through the assessment of the individual successfulness, the management can obtain information about the units where the employee acts.

The system of assessment of successfulness provides information to managers, related to the skills and the abilities of the employees, and with a quality supervision. If this system is successfully implemented, sufficient required information is provided to the management for analysis of the role for effective successfulness and for the development of the employees $[1,2]$.

The assessment process is not simple to implement, because it includes a debate on the efficiency of the employees, criticism and praise is accepted, they indicate the strengths and the weaknesses, and in some cases also a recommendation of some of the employees to build their future somewhere else.

\section{Methods of Assessments}

The work efficiency is usually assessed from the existing assessment methods. In many types of work, they are usually the only way to distinguish and assess the work efficiency in both employees and managers. For this purpose, many methods and assessment techniques have been developed. They are distinguished by the level of elaboration, the ways how they are applied, the criteria for assessment, some rather emphasize the general properties, the work behavior, the results, etc. The most famous assessment methods are: the comparative method, scales of assessment, and "check-list" [3,4].

\section{Comparative method}

The comparative assessment method is a method which compares the individual to the other employees and it can be reviewed through the following methods: ranking, comparison to pairs and forced distribution.

Ranking is simpler and oldest method which requires the managers to rank the members of their group according to the efficiency in work, starting from the highest to the lowest rank. The ranking procedure is relatively simple; however, the assessor may have difficulties in ranking the associates in the middle part, while it is easier to separate the ones who are best and weakest.

Comparison to pairs: This is a method which requires from the assessor to systematically compare every employee to another one in the other group (department, enterprise) according to the total work efficiency. In each pair, the assessor must decide which of the members is better. The assessment is performed for one or several characteristics.

Forced distribution: This is a method of group ranking which can be useful in the following examples:

- An assessor should assess many associates

- Rough differentiation is sufficient

- One can assume that the distribution of work efficiency of the associates corresponds to the normal distribution.

The task of the appraiser is to arrange the assessed in several categories for work efficiency, whereby every category will have an advanced determined percentage of the ones which can be classified in the category $[5,6]$.

The assessor assesses the employees within fixed distribution between the levels of performances; $10 \%$ unsatisfactory, $20 \%$ sufficient, $40 \%$ satisfactory, $20 \%$ good, and $10 \%$ excellent. One of the reasons why this technique was created is to assess problems such as excessively good grades or when all employees are averagely assessed. On the other hand, if all employees are assessed as excellent, this means that the evaluation system was not properly used.

It is assumed that only a relatively small percentage of people work

*Corresponding author: Nermin Ademi, Department of Business and Economics, Ministry of Culture, Macedonia, Tel: 02/3240-555; 3240-500; E-mail: n.ademi@kultura.gov.mk

Received July 20, 2017; Accepted September 22, 2017; Published September 30, 2017

Citation: Ademi N (2017) Programming and Implementation of Assessment. J Bus Fin Aff 6: 291. doi: 10.4172/2167-0234.1000291

Copyright: () 2017 Ademi N. This is an open-access article distributed under the terms of the Creative Commons Attribution License, which permits unrestricted use, distribution, and reproduction in any medium, provided the original author and source are credited. 
significantly above the average, the percentage of unsuccessful people is low, while everyone else is somewhere in the middle (Figure 1).

The advantage of the comparative method compared to the other methods is that by forcing the assessors to compare the associates who assess, and then they eliminate the emergence of standard errors of the assessor (the gentleness, the strictness and the central tendency).

\section{Scales of the assessment}

This assessment method belongs to the category which assesses the individual efficiency compared to some previously established work standards, that is, criteria on the work success.

Usually two reasons are pointed out for its popularity:

- It is relatively easy to construct it

- They try to be increasingly objective.

According to the scale of assessment, there are two basic groups: graphical and descriptive, that is, descriptive scales.

Graphic scales: It is more frequently applied as an assessment method. The simplest example of this method is school assessment of five numerically expressed levels. They can be continuous and discontinuous, that is, they may have many levels, and it is possible to use different characters, lines, verbal designations, that is, adjectives (bad, above-average, average, below-average, excellent) (Figure 2) [7,8].

Descriptive, that is descriptive scales: This is a method wherewith instead of a number of some other attributes, short or detailed description is provided in every dimension of the efficiency. There are numerous variants and they differ according to the elaboration in the description of the behavior which denotes bad or good work efficiency, which enables the assessors to more easily and more objectively detect and determine on the specific work behavior. Many enterprises assess that, with the application of the descriptive scale, they are able to learn as much as possible about the level of competence of their members, such as the professional, the operational, the interpersonal competence, etc. The advantage of this method of assessment is that it is relatively simple for application, only a scale for all employees or for the key categories of employees can be prepared (for example, for managers

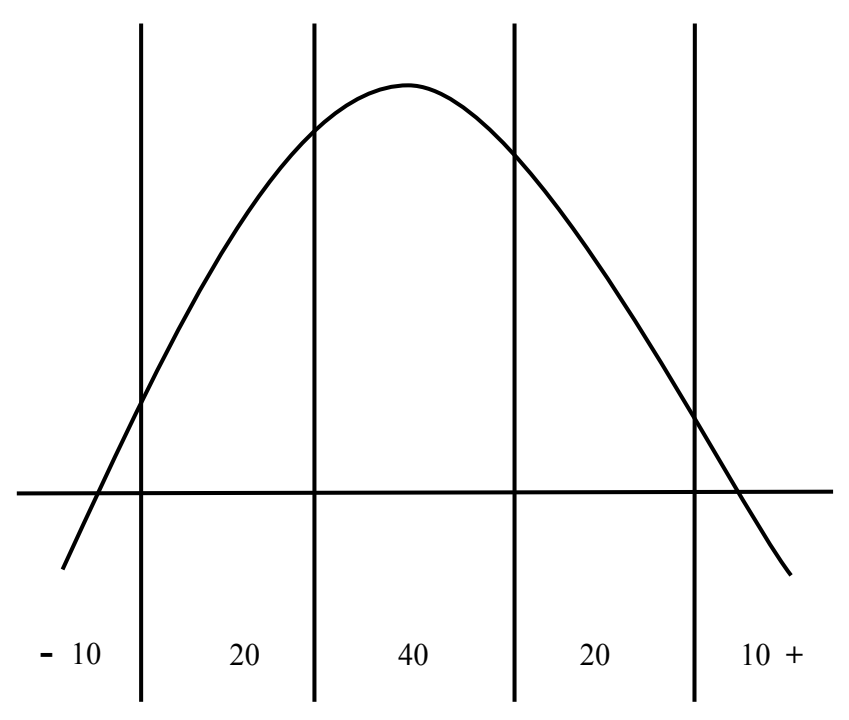

Figure 1: Compulsory distribution.

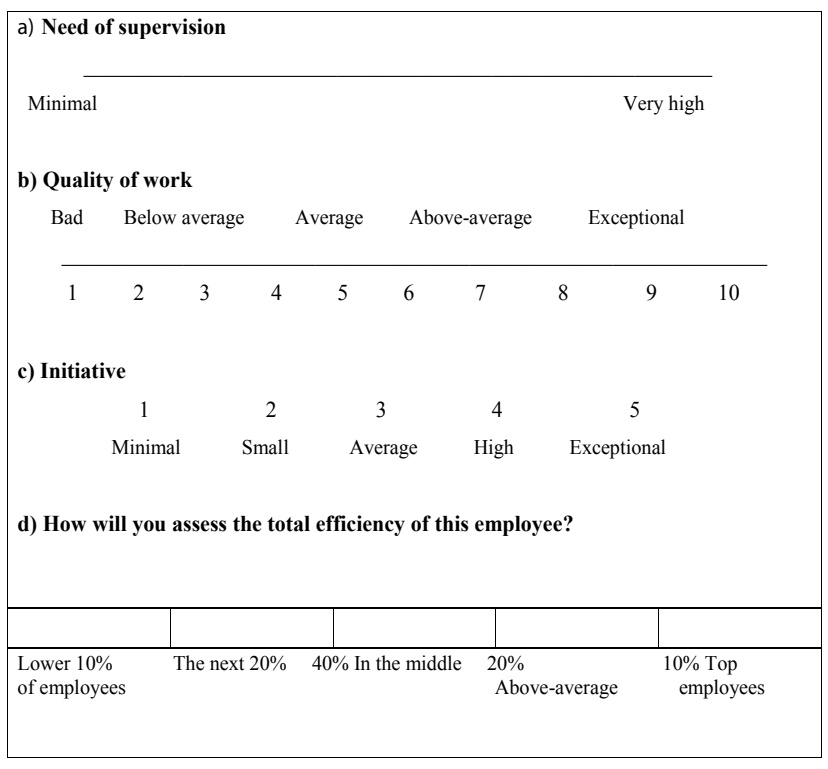

Figure 2: Graphic scale.

and non-managers) and specific indicators in the total work efficiency can be quantitatively expressed.

\section{“Check-list”}

In the attempt to overcome the deficiencies in the assessment methods, another method emerges, called a "check-list", which comprises of a sequence of specific reports which describe the different forms of behavior in the specific work. The specific behavior at work is observed through a sequence of positive and negative reports, while the assignment of the assessor is to mark the ones which mostly correspond or do not correspond to the work behavior of the person who is being assessed.

There are two basic variants of this technique: a list of free choice and a list of forced choice. A list of free choice makes several reports which describe the work behavior as important for successful or unsuccessful performance of the specific work, while the task of the assessor is to freely choose, that is, to mark the ones which best match the work behavior. The reports by default describe the positive and the negative behavior. The behaviors which are being described can be unweighted (which means that every positive report brings, for example, one positive point, while the negative report brings one negative point), and weighted (which means that they should have a different weight which if determined in advance, that is, the number of positive and negative points they bear).

The list of forced choice tries to objectify the assessment process and to eliminate the subjective mistakes of the assessor. The assessor must choose in every specific group who suits him the most and who suits him the least for the description of the behavior of the specific person. Positive scoring is performed in situations when the assessed person corresponds the most and describes the work behavior best, has positive valorized report, while the person gets negative points in a contrary situation.

This method is considered a relative objective instrument for measurement of success. Very often its advantage is highlighted as one 
of the greatest problems, which is the discontent of the assessor why they do not have a control over the actual results of their assessment.

One of the most important reasons for its relatively rare use is the fact that the list should be constructed for a specific work, that is, a specific job position and the construction procedure is very complex, and in terms of its use it is a very expensive technique.

\section{Organizational set-up in the assessment}

Who is invited to assess: In regard to the assessment, there are different possibilities, some of which are more frequently used in practice, while others are rarely used. The assessment requires direct knowledge and direct observation of the work behavior. An assessor can be everyone who monitors and knows this behavior. The successfulness of an assessor depends on his ability to successfully observe, and of course, to be able to transform these observations into grades. Therefrom, one can conclude that the assessors can be: superior managers, associates and colleagues, subordinates as well as the person who is being assessed (self-assessment). Recently in some enterprises, customers and clients are also assessors [9].

Superior managers: Usually, the managers are traditional assessors of work efficiency. Usually, they are superiors who by default constantly observe the work efficiency and the successfulness of their associates during work. Research has shown that managers include more than 95\% in the assessment programs in industry. Also in cases when the assessment is performed by other assessors, the manager also assesses the work efficiency, and then a final assessment is obtained based on several assessors [10].

Associates and colleagues: This assessment group usually includes the members of the working group who can be the best experts of some matter. Sometimes it is also called mutual assessment, because every member of the group assesses all the other members (apart from himself/herself). Sometimes, they give information which cannot be obtained from the managers. However, in the enterprise, there is doubt in the assessment by the colleagues for many causes. First, the fear that the colleagues will be very mild. Second, the concern that it may cause competence and mistrust between the colleagues, that is, the associates. Some research has shown that the assessment by the colleagues is prone to prejudices, such as racial or other prejudices. However, it turned out that the assessment by the colleagues is a good prognosis for the future work efficiency. Its advantage is that it has several assessors and it increases the objectivity in the assessment.

Self-assessment: Recently self-assessment is increasingly being used for assessment. It is considered that the participation of the employees in the assessment process improves their motivation and reduces the defensive attitude during the conversations on efficiency [11].

However, it turned out that self-assessment tends to be milder, less variable and usually slightly matches the assessment by the others. In practice, managerial behavior is used. The manager and the person who assesses performed a separate assessment of the efficiency as a basis for a mutual dispute and coordination of the mutual differences. This procedure is increasingly applied in the enterprises.

Subordinates: Usually the subordinates assess some dimensions of the managerial efficiency such as team work, communication, quality, leadership, planning and development of the associates. These dimensions such as know-how, administration and innovations better assess the subordinates.

This assessment is also called "upward" assessment (companies which apply it include Chrysler, IBM, AT\&T, etc.) and it provides managers a lot of useful information which help them to promote their style of management [12].

Consumers and clients: This is one way to get an assessment from the external consumers and clients through specialized questionnaires, telephone interviews and other standard techniques where the tendency is to get an opinion about the efficiency of a specific employee, sector or enterprise, about the opinion they share on their work efficiency. An increasing number of enterprises use "the customer satisfaction with the service" as a basis for stimulating awarding. This technique can also be used for valuation of educational programs and processes, determination of the educational needs and other goals.

\section{Determination of the time frame}

The time frame in assessment is a very important factor for its successful implementation. The assessment should be based on cooperation between the two parties, therefore, it is desirable to choose the time which is appropriate to the parties in the process. This period should be a relatively peaceful period of the year or the month, avoiding the chaotic moments at the end of the month, the year, after the holidays or annual leaves.

The determination of the time for assessment also implies the dedication for preparation and its participation, whereby its delay for no particular reason may cause resistance and negative signal for the values which are assigned to the assessment process. According to Hovard Hudson, at least two hours should be provided for complete appropriate assessment of a company's employees. If the assessment should exceed this time frame, it is better to terminate the assessment and to continue on some other day. In long processes, it is more efficient to dispute in depth about some issues, than only to start many issues without resolving any in a satisfactory way.

When someone new or someone who has recently been repositioned among the employees, is assessed for the first time, it is obvious that more matters have to be covered about this person than for someone who has been assessed for several years. Increased dedication to the new employees and the creation of good relations is an investment which will pay off in the future $[5,9]$.

The frequency when the employees should be assessed depends on the complexity of their work and their abilities. Everyone should have one comprehensive assessment at least once a year, in order to check "the current status of the person, his/her course and how he/she will succeed further on". These assessments will probably be sufficient for the employees who perform simple works and who have experience. For newly employed or for someone who has been promoted recently and undertakes new responsibilities, in the beginning a monthly assessment should be performed, sometimes even in weekly intervals. Such careful observation can prevent eventual problems and it can strengthen the ability to work in the new field, and also this builds trust in the very process of assessment.

\section{What is valued?}

When assessing the efficiency of work, one should consider the following facts: how the worker performs the work, what the results are, how well he/she performed the work assignments. Some results are measurable, while in many types of works it is impossible to measure the results. Furthermore, the measurement of the results does not provide complete image, even if it reduces the motivation and the satisfaction of the employees. The behaviors can show whether the bad 
results are a reflection of the inadequate skills of the employees, the lack of effort or poor working conditions. Usually the behavior and the results are measured.

In the assessment, one should not reflect only the way how the employees perform the work assignments, but also their business relations, as well as their strives for their career. When goals are being determined, one should always have in mind only the outcome, and not the possible ways to them. Therefrom, it arises that the employees should not be given only an assignment, but also responsibility.

Many managers are concentrated on the uppermost and the lowest point of performance in the assessment. However, it would be more useful if attention was paid to what is fine at the moment and the focus should be on its improvement. Almost $90 \%$ of the time for assessment is wasted in a dispute for the current and the past performance, while $10 \%$ of the conversation is dedicated to the future. Although an important lesson can be learned from the past success or failure, still the most positive results would be achieved if that discussion is an introduction for improvement of the future.

\section{Some problems and their resolution during the assessment}

In the literature of countries which deal with assessment of successfulness for years, there is criticism about the different methods. The reasons for the criticism usually arise from the unpreparedness of the assessors or from the fact that the instruments for description of the job positions are not closely related to the purpose of the assessment.

Both problems undoubtedly emerge as soon as the enterprise starts with systematic assessment of the employees. With the intention to avoid the mistakes which can objectively be avoided, we will mention the most common ones:

- Lack of objectivity is a traditional problem of every assessor. After ranking the individual characteristics of the employees, general attitudes emerge, loyalty to the enterprises, the characteristics of the person who assesses, etc. There will always be specific subjectivism, however, quality questionnaires and training for the assessor are required in order to reduce the subjectivism.

- Halo effect occurs when the assessor underlines one (positive or negative) characteristic of the employee and estimates it more (for example: neatness), whereby this affects all the others.

- The prejudices of the assessor such as the treatment of males and females, years of age, religion or nationality can negatively affect the objectiveness of the assessment. Indeed, it seems that it is most difficult to fight these; however, they must be mitigated with the increasing number of specific defined characteristics which are assessed with grades 1-5 or 1-10.

Central tendency is an error according to which everyone who assesses is placed in a space of averageness, with a grade "average" that is (with the number) 3 if the scale comprises of five levels. If in some of the managers, many average grades emerge, the assessment should be changed or it should be repeated upon a request.

\section{Conclusion}

Despite of the numerous mistakes which the assessors can make in the assessment, there is a sequence of possibilities for their improvement although the human can never be a perfect "measuring instrument". However, his responsibility and objectivity in the assessment improves.
There are several ways to improve the business success in the assessors such as:

- Training of the assessor

- $\quad$ Provision of feedback

- Use of several assessors

- Horizontal assessment

- Improvement of the methods of assessment.

These techniques can improve the quality of assessment, however, unfortunately this can never make it objective and accurate, and it cannot eliminate the need to speak about the subjective methods of assessment.

\section{References}

1. Mount MK (1984) Psychometric Properties of Subordinate Ratings of Managerial Performance. Personnel Psychology 37: 687-702

2. Joiner CL, Hyde JC (1998) Performance appraisal.

3. Waxley KN, Yukl GA (1984) Organizational Behavior and Personnel Psychology, (2nd edn.), Homewood, IL, IRWIN, p: 570.

4. Sveto M (2006) Human Resource Management (Upravljanje Ljudskim Potencijalima) - Zagreb.

5. Schultz DP, Schultz SE (1986) Psychology and Industry Today: An introduction to industrial and organizational psychology. Macmillan, New York.

6. Borman WC (1991) Job Behavior, Performance and Effectiveness. Dunnette MD, Hough LM editors. Handbook of Industrial and Organizational Psychology, CA, pp: 271-326.

7. Dipboye RL, Smith CS, Howell WC (1994) Understanding Industrial and Organizational Psychology: An Integrated Approach. Forth Worth, TX, Harcourt Brace Coll. Pub.

8. Oppler SH, Campbell JP, Borman WC (1992) Three Approaches to the Investigation of Subgroup Bias in Performance Measurement: Review, Results and Conclusions. Journal of Applied Psychology 77: 201-217.

9. Campbell DJ, Lee C (1988) Self-Appraisal in Performance Evaluation: Development versus Evaluation. Academy of Management Review 13: 302-314.

10. Harris MM, Schaubroeck J (1988) A Meta-Analysis of Self-Supervisor, SelfPeer and Peer-Supervisor Ratings. Personnel Psychology.

11. Naumovska L (2008) Human Resource Management. ( $2^{\text {nd }}$ edn.), Skopje: European University - Republic of Macedonia.

12. Howard H (1992) The Perfect Appraisal: All you need to get it right first time Random House. 